\section{PARTICIPAÇÃO DE ADOLESCENTES EM AÇÕES EDUCATIVAS SOBRE SAÚDE SEXUAL E CONTRACEPÇÃO}

\author{
Participation of teens in educational actions on sexual health \\ and contraception
}

\section{RESUMO}

Objetivo: Descrever a participação de adolescentes em ações educativas sobre saúde sexual e contracepção. Métodos: Estudo descritivo, com pressupostos da pesquisa-ação, desenvolvido com 30 adolescentes do $8^{\circ}$ e $9^{\circ}$ anos em Escola do Ensino Fundamental, em Fortaleza, Ceará, Brasil. A coleta de dados foi realizada de forma coletiva em rodas de conversas e oficinas educativas sobre gravidez e contracepção na adolescência em três encontros entre os meses de junho e novembro de 2015. As informações foram analisadas abstraindo-se os temas e núcleos de sentidos, os quais agrupados na categoria: Prevenção da gravidez na adolescência; Conhecimento sobre uso dos métodos contraceptivos. Resultados: Apresentam os significados das mensagens dos adolescentes que expressaram conhecimentos, dúvidas e curiosidades geradas nas conversas e oficinas sobre prevenção da gravidez na adolescência. Destacaram-se os principais métodos contraceptivos utilizados pelos adolescentes como o preservativo masculino e a pílula do dia seguinte, porém apresentaram muitas dúvidas e questionamentos sobre o uso adequado. Conclusão: O modo criativo e participativo de abordar o tema favoreceu esclarecimentos, redução de inquietações e autorreflexão sobre a prevenção da gravidez e autonomia dos escolares ante a escolha dos contraceptivos.

Descritores: Adolescente; Contracepção; Educação em Saúde.

\section{ABSTRACT}

Objective: To describe the teenager participation in educational interventions on health and sexual contraception. Methods: A descriptive study, with assumptions of action research, developed with 30 adolescents of 8 th and 9 th grade, in Middle School in Fortaleza, Ceará, Brazil. Data collection was carried out collectively on wheels talks and educational workshops on pregnancy and contraception in adolescence in three meetings, in the months of june and november of 2015. The information was analyzed abstracting the themes and core senses, which grouped in category: Prevention of teenage pregnancy; Knowledge about the use of contraceptive methods. Results: Bring the meanings of the messages adolescents who expressed knowledge, doubts and curiosities generated in talks and workshops on prevention of teen pregnancy. The highlights were the main contraceptive methods used by adolescents as the male condom and morning-after pill, but had many doubts and questions about the proper use. Conclusion: The creative and participatory way to address the issue favored clarification, reduction concerns and self-reflection on the prevention of pregnancy and autonomy of school at the choice of contraceptives.

Descriptors: Adolescents; Contraception; Health Education.
Artigo Original
1) Universidade Estadual do Ceará - UECE - Fortaleza (CE) - Brasil

2) Universidade de Fortaleza - UNIFOR Fortaleza (CE) - Brasil
Este artigo seguiu as normas e formatação estabelecidas pelo $5^{\circ}$ CIAIQ - Congresso Ibero-Americano em Investigação Qualitativa. 


\section{INTRODUÇÃO}

Adolescência, fase da vida entre 10 e 19 anos de idade, é marcada pelo rápido desenvolvimento, mudanças biológicas, psicológicas e sociais. Período da maturação sexual e aquisição da capacidade de reprodução, favorecendo o desenvolvimento de uma identidade adulta ${ }^{(1,2)}$.

Ao mesmo tempo ocorrem as mudanças biopsicossociais e a vulnerabilidade própria da fase. Os riscos físicos, emocionais e sociais a que se expõem mostram a prática sexual não segura, com exposição à infecção por doenças sexualmente transmissíveis DST/AIDS, a gravidez na adolescência e outros, que foge do controle no âmbito individual $^{(3,4)}$. Portanto, a atividade sexual precoce tem repercussões na vida do adolescente, em decorrência da exposição desses jovens aos riscos da gravidez não planejada e das DST ${ }^{(5)}$.

Em relação à gravidez na adolescência, discutem-se os aspectos que favorecem o aumento desta ocorrência, destacando-se o início precoce da vida sexual, associado ao desconhecimento do uso de métodos contraceptivos, dificuldade de acesso a programas de planejamento familiar, desconhecimento slobre as complicações e consequências que a gravidez pode acarretar, incluindo a desestruturação familiar ${ }^{(3)}$.

A gravidez na adolescência deve ser abordada de forma ampliada focando-se o aspecto da sexualidade, sem julgamentos de valores e com respeitos aos direitos sexuais e reprodutivos de adolescentes e jovens. Há necessidade de uma abertura dos canais de comunicação com os adolescentes, principalmente na escola, família e comunidade, contribuindo assim para o fortalecimento da autonomia e do autocuidado ${ }^{(6)}$. Ao desenvolver educação em saúde sexual com os adolescentes, os profissionais devem direcionar ações com o intuito de atender suas necessidades e fortalecer autonomia para a promoção da sua saúde ${ }^{(7)}$.

Desse modo, ao abordar adolescentes sobre sexualidade e contracepção, faz-se necessário envolvê-lo em estratégias de aprendizagem que facilite a troca de informação e a aquisição de saberes. O ideal é que o educador estimule o debate sobre atitudes para promoção da saúde, ajudando o adolescente a decidir sobre práticas seguras, exercendo assim sua sexualidade de forma segura e responsável ${ }^{(8)}$. Nesse contexto, a sexualidade e a contracepção devem ser tratadas de forma multidisciplinar e interdisciplinar, famílias, escolas, comunidades, instituições que reúnem adolescentes e, até mesmo, nos locais de lazer, para que eles vivenciar esta situação com mais segurança. O debate sobre sexualidade e contracepção com os adolescentes favorece a apreensão de mais conhecimento sobre as DST, as vulnerabilidades da gravidez na adolescência como também sobre as políticas públicas que regem o cuidado a esta faixa etária ${ }^{(9)}$.

A Política Nacional de Atenção Integral à Saúde de Adolescentes e Jovens no Brasil assume o objetivo de incorporar a atenção deste grupo populacional à estrutura e aos mecanismos de gestão, à rede de atenção do Sistema Único de Saúde (SUS) e às ações de rotina destes, em todos os níveis ${ }^{(10)}$. Defende, portanto, a utilização de estratégias educativas mais aceitas por essa clientela. Assim, educadores e profissionais de saúde devem promover espaços para discussões das temáticas sexualidade e contracepção com adolescentes, de modo que reflexão e sensibilização possam esclarecer dúvidas; proporcionar conhecimentos e motivações que contribuam para a formação de pessoas com competências nas escolhas e decisões responsáveis ${ }^{(11)}$.

Neste aspecto, observa-se a necessidade de fortalecer as ações de educação em saúde nas escolas, pois esta é um ambiente adequado à promoção da saúde ${ }^{(10)}$. Atualmente, o Programa Saúde Escolar (PSE), tem como finalidade fortalecer a articulação de ações entre as escolas públicas e a Estratégia de Saúde da Família, favorecendo a promoção da saúde, o que sugere a ideia de partilhar conhecimentos, compromissos, significados, valores, responsabilidade e ações ${ }^{(12)}$. Por meio de estratégia intersetorial, a saúde e a educação foram planejadas de forma mais integradora, mas há necessidade de um trabalho contínuo com os adolescentes nestes ambientes utilizando-se abordagens que potencializem a autonomia dos sujeitos e o livre exercício da cidadania ${ }^{(13)}$.

A implementação de estratégias educativas com esforços conjuntos de educadores e profissionais da saúde, tem dentre os objetivos a prevenção de DST/HIV/AIDS e gravidez na adolescência ${ }^{(14)}$. Deste modo, a integração entre os profissionais da atenção primária e da escola no desenvolvimento de ações educativas com os adolescentes podem produzir novas maneiras de pensar e agir na saúde sexual e reprodutiva favorecendo a redução de riscos e a promoção da saúde desta população. Por conseguinte, é necessária uma aproximação com a realidade dos adolescentes, incluindo seus saberes e desenvolver ações educativas com participação ativa dos mesmos, essencialmente no ambiente da escola, vislumbrando práticas sexuais seguras e prevenção da gravidez não planejada.

Diante do exposto, o estudo foi conduzido pelos questionamentos: como ocorre a participação dos adolescentes em atividades educativas sobre os métodos contraceptivos? A pesquisa é recorte de um projeto "Promoção da saúde dos adolescentes na escola" e seguindo os pressupostos da pesquisa-ação, efetivaram-se atividades educativas e os procedimentos investigativos 
envolveram reflexões entre pesquisadores e adolescentes sobre a temática saúde sexual e contracepção. À luz desse referencial e para responder à questão da pesquisa delineouse como objetivo: descrever a participação de adolescentes em ações educativas sobre saúde sexual e contracepção.

\section{MÉTODOS}

Estudo descritivo, utilizando pressupostos da pesquisaação, portanto participação ativa dos sujeitos em ações educativas com momentos de reflexões e, ao mesmo tempo, elaboração de conhecimentos sobre gravidez na adolescência e métodos contraceptivos.

A pesquisa-ação parte de um trabalho da coletividade em que todos os atores envolvidos são pesquisadores e/ou participantes atuando ativamente ou de forma cooperativa buscando encontrar possíveis soluções para a situação ou problema que estão enfrentando ${ }^{(15)}$. Assim, se utilizou métodos participativos como oficinas educativas para uma melhor aproximação com o objeto na busca de informações e reflexão-ação junto aos adolescentes.

A pesquisa e as intervenções educativas foram realizadas com a presença de dois facilitadores (bolsistas da graduação de enfermagem) e um docente com 30 alunos do $8^{\circ}$ e $9^{\circ}$ anos, incluídos a partir da frequência regular na escola e a disponibilidade em participar das atividades planejadas. Promoveram-se grupos educativos semanais, intercalados nas atividades de aula, horários concedidos pelos professores e direção da Escola Pública Municipal Projeto Nascente, no município de Fortaleza, CE, Brasil entre junho e novembro de 2015. As atividades grupais com oficinas e dinâmicas tinham a finalidade de sensibilizar, utilizar a informação e a reflexão como proposta de aprendizagem compartilhada sobre o tema em discussão(16).

As atividades de pesquisa e investigação ocorreram em três encontros, cada com duração de 50 minutos e as ações educativas tiveram a participação e concordância dos gestores e professores da escola. O primeiro encontro foi pautado em conversas e evocação da temática "gravidez na adolescência" com elaboração de desenhos e histórias que representavam conhecimentos dos adolescentes no assunto. No segundo encontro promoveram-se espaços de conversas para que os adolescentes discorressem livremente o que pensavam sobre "métodos contraceptivos". No terceiro encontro houve um aprofundamento da temática: métodos contraceptivos com apresentação do mostruário de alguns métodos (preservativo masculino e feminino, DIU, diafragma, anticoncepção de emergência, espermicida, implante subcutâneo, anticoncepcional oral e injetável). As temáticas discutidas foram amplamente debatidas pelas pesquisadoras dirimindo as dúvidas e estimulando outras perguntas relacionadas aos assuntos explorados na dinâmica mitos e verdades.

Dessa forma, os adolescentes estimulavam-se a falar em todos os momentos da construção coletiva e as informações foram gravadas em áudio por um celular do modelo Samsung, transcrito por uma das autoras, além de utilizar-se do diário do pesquisador. A composição do corpus envolveu os registros das atividades educativas com os adolescentes e utilizou-se a análise de conteúdo ${ }^{(17)}$. Abstraíram-se os temas e núcleos de sentidos, que foram agrupados nas categorias: Prevenção da gravidez na adolescência; Conhecimento sobre uso dos métodos contraceptivos.

A investigação respeitou os preceitos éticos da Resolução $n^{\circ}$. 466/12 ${ }^{(18)}$, recebeu anuência da instituição e dos participantes por meio da assinatura do Termo de Consentimento Livre e Esclarecido (TCLE) pelos pais e/ ou responsáveis e do Termo de Assentimento Livre e Esclarecido (TALE) pelos adolescentes. O projeto adquiriu parecer de aprovação do Comitê de Ética da Universidade Estadual do Ceará, conforme n ${ }^{\circ} 651.771$.

\section{RESULTADOS E DISCUSSÃO}

\section{Prevenção da gravidez na adolescência}

Para aproximar-se da realidade e dos conhecimentos dos adolescentes iniciaram-se as conversas grupais e a construção de desenhos e histórias representando a atividade sexual e a prevenção da gravidez ilustrados nos discursos/ síntese.

"Tenha responsabilidade e use camisinha... Sexo pode ser bom, mas se previna, para não engravidar e nem pegar doenças, como a HIV [...]."

"Tomar remédio, usar camisinha, tomar injeção... para que você não sofra as consequências lá na frente, pois tem a vida toda para curtir [...]."

"Cuidados na gravidez! Para se prevenir: camisinha masculina, pílula do dia seguinte, camisinha feminina, espermicida, tabelinha."

Só engravida quem quer por que tem como se prevenir: injeção, pílula do dia seguinte, camisinha unissex, anticoncepcional [...]."

Os adolescentes, ao pensarem em atividade sexual associaram à gravidez e aos principais métodos contraceptivos que conhecem ou aqueles acessam em suas experiências, sendo o preservativo masculino e a pílula do dia seguinte o mais comentado em suas conversas. Também sinalizaram que não usam os métodos contraceptivos corretamente. Os resultados encontrados neste estudo estão em consonância com outras pesquisas que identificaram como métodos mais usados pelos adolescentes a camisinha 
e os anticoncepcionais orais e injetáveis, enquanto a tabelinha, Dispositivo Intrauterino (DIU) e espermicida são métodos menos conhecidos pelos adolescentes ${ }^{(13,19)}$. Outro estudo observou o não uso do preservativo ou uso incorreto e alertam sobre os riscos à saúde, além da gravidez precoce $^{(20)}$.

É reconhecido que a escola tem papel fundamental no desenvolvimento do adolescente, contribui com a formação global do jovem e da sociedade. Papel que extrapola o ato de ensinar e envolve o educar crianças e jovens desenvolvendo sua identidade e subjetividade ${ }^{(14)}$. Na atividade educativa com os adolescentes, os facilitadores promoveram espaços de conversas que lhes permitiram discorrer livremente e refletirem sobre as possibilidades existentes de prevenção da gravidez. O espaço para o diálogo entre colegas da escola sobre a saúde sexual é comum, porém em atividades educativas neste espaço é raro, pois nem sempre os educadores dispõem tempo para esta atividade nas aulas. A ausência de diálogos sobre a temática estudada tem prejudicado reflexões e atitudes quanto ao exercício de práticas sexuais protegidas. Percebe-se que é uma temática que ainda está arraigada de preconceitos, pais e professores parecem conversar pouco com os adolescentes para que eles exerçam com segurança sua sexualidade. Contudo, o tema da saúde está inserido nos Parâmetros Curriculares e devem ser inseridos de forma transversal e serem abordados em conteúdo do Ensino Fundamental ${ }^{(12)}$.

Ressalta-se a importância de programas governamentais para fortalecer a temática saúde sexual e reprodutiva dos adolescentes escolares e para que os setores saúde, educação e família em conjunto possam orientá-los quanto à gravidez precoce, prevenção de DST/AIDS e sexualidade, visando à minimização desses agravos ${ }^{(21)}$.

Recomenda-se que, nas conversas com os adolescentes, deve-se incluir não apenas a oferta de preservativos feminino e masculino, como também os demais métodos contraceptivos, proporcionando espaço para que o adolescente possa falar de si, trocar experiência e apreender informações corretas que favoreçam a adoção de hábitos de vida saudáveis. Notadamente, o número de adolescente que inicia a atividade sexual está mais evidente e precoce e, muitas das vezes, pode estar associada ao desconhecimento dos métodos anticoncepcionais e da reprodução ${ }^{(22)}$.

A gravidez na adolescência passou a ser vista como uma situação de risco biopsicossocial, capaz de trazer consequências negativas não apenas para as adolescentes, mas para toda a sociedade. Tornou-se, por isso, um problema social e de saúde pública ${ }^{(23)}$. A oficina sobre educação sexual e contracepção na gravidez propõe oferecer ao adolescente, momentos de reflexão e construção dos saberes, como sujeito ativo no processo de maturação pessoal. A educação sexual e reprodutiva deve incluir vários atores sociais e incluir os jovens de um modo geral e não somente as adolescentes mulheres. Quando há oficinas de educação sexual e reprodutiva, o conhecimento que é repassado para os alunos é muito superficial, o que acarreta muitas dúvidas na utilização dos métodos contraceptivos, e na maioria das vezes, não há, por parte dos educadores um trabalho contínuo para mostrar as consequências de uma prática sexual insegura ${ }^{(24)}$.

Neste sentido, assinala-se que quando ocorre a gravidez, entre adolescentes, embora não seja planejado, eles expressam sentimentos, relacionados ao papel de pai e de mãe. Esta na responsabilidade de cuidar do filho, enquanto o pai tem a preocupação de prover o sustento da família. Observa-se que culturalmente, a mulher sempre assume mais responsabilidades e na descoberta da gravidez, a adolescente atravessa uma mudança na sua rotina de estudos, de trabalho e de lazer, assumindo maiores responsabilidades do que o homem ${ }^{(25)}$. Assume responsabilidades que antes não tinha e nem sempre dar continuidade aos estudos, ou seja, há ruptura dos projetos pessoais, afastamento da escola e das interações sociais em decorrência da gravidez. Por isso, é relevante o apoio da família, escola e dos profissionais de saúde, para que os adolescentes possam lidar com esta situação com mais segurança ${ }^{(21,26)}$.

Logo, tem-se a necessidade de investir esforços no intuito de prevenir a gravidez na adolescência e não excluir o adolescente, pois poderá também ter consequência da gravidez precoce. Nesta perspectiva, o espaço oferecido aos adolescentes de ambos os sexos, em rodas de conversas e/ ou oficinas, supõe-se como oportunidades de autorreflexão sobre questões da prevenção da gravidez.

\section{Conhecimento sobre uso dos métodos contraceptivos}

Neste estudo, além de discutirem sobre a prevenção da gravidez, houve afirmações sobre a prevenção de Doenças Sexualmente Transmissíveis (DST), situações problematizadasporeles, quandoinstigadosase expressarem, por exemplo, muitos adolescentes responderam que as DST eram transmitidas através do beijo. E sabe-se que não é verdade. $\mathrm{O}$ único método contraceptivo que protege contra as DST são os preservativos masculino e feminino. Entretanto o número de assertivas referente aos alunos do $9^{\circ}$ ano quando se questionou o uso da pílula e do preservativo para evitar gravidez e DST foi maior em relação ao $8^{\circ}$ ano. Pode-se inferir a partir disso que os alunos do $9^{\circ}$ ano, como fazem parte da adolescência maior, os profissionais falam do assunto com eles, pois o capítulo de corpo humano é aprofundado neste período, portanto há possibilidades de conversar sobre os órgãos reprodutores e planejamento reprodutivo, dentre outros assuntos relacionados ${ }^{(11)}$. Outro comentário entre os alunos maiores foi sobre a pílula do dia 
seguinte, conhecida pela maior parte dos alunos, porém não sabem sobre a sua utilização corretamente.

A atenção aos adolescentes no sistema de saúde e educação vem sendo discutida na perspectiva da abordagem multiprofissional, com vistas a melhorar o cuidado a esses jovens, que ainda tem poucas oportunidades de desenvolverem atitudes saudáveis ${ }^{(10)}$. Ressalta-se que somente passar a informação ao adolescente acerca dos métodos contraceptivos existentes, não é suficiente, mas deve-se ampliar o conhecimento sobre a importância de alguns métodos na prevenção de doenças sexualmente transmissíveis, o efeito de cada um no corpo, a forma correta de sua utilização e as possíveis consequências do não uso $^{(22)}$. Desse modo, comprometer-se com a saúde e ampliar a responsabilidade dos adolescentes pode ampliar a autonomia para escolhas conscientes. Assim a educação sexual é uma responsabilidade compartilhada entre educadores, profissionais de saúde, além dos pais que podem ter esses diálogos quando se sentem à vontade e aptos a conversarem com seus filhos sobre $\mathrm{o}$ assunto.

Os adolescentes confirmaram ideias sobre a prevenção da gravidez, trazendo informações sobre o uso dos contraceptivos, do senso comum ou mesmo das conversas com os profissionais.

"O uso da camisinha previne a gravidez e as doenças sexualmente transmissiveis [...].'

"Eu acho que a pílula do dia seguinte se toma quando tem uma relação sexual sem camisinha e o homem... ejacula dentro da mulher, ai ela toma dentro das primeiras 24 horas [...]."

"Eu acho que a pílula não pode se esquecer de usar, mas não protege contra as DST [...].”

"Eu já ouvi falar que a camisinha masculina é que previne a gravidez e as DST, se usada corretamente, ela previne a gravidez [...]."

São muitas as razões para comportamentos sexuais desprotegidos entre adolescentes e, sem dúvida, o desconhecimento sobre o próprio corpo, os métodos contraceptivos e seu uso correto têm forte evidência. Outros estudos reforçam que a aplicação do conhecimento é uma forma de prevenir a gravidez na adolescência, evitando que o futuro da adolescente seja comprometido ${ }^{(26,27)}$.

Pesquisa realizada em Minas Gerais, Brasil, constatou que os adolescentes consideram a prevenção da gravidez na adolescência como algo positivo e importante, porém apontaram que o sexo inseguro e as dúvidas dos métodos anticoncepcionais perduram juntamente com as falhas dos profissionais de saúde ${ }^{(28)}$.

No terceiro encontro com os adolescentes, foram retomadas as discussões sobre os métodos contraceptivos.
Os participantes eram encorajados a discutir, além dos tipos de contraceptivos o seu uso correto. Assim, a conclusão da atividade educativa teve o intuito de elucidar a principal temática de interesse dos adolescentes - os contraceptivos reforçando-se aqueles mais usados entre eles e reafirmando o acesso nas unidades de saúde. Neste sentido, defendese que o adolescente necessita de olhar diferenciado e, às vezes, individualizado ${ }^{(22)}$. Embora se percebam questões socioculturais do grupo, é preciso ouvir o que cada um tem a dizer, a subjetividade, procurando não coletivizar tudo, mesmo nas ações grupais.

Nessa dinâmica, ao discorrer sobre cada método, estabelecia-se uma relação de troca de saberes com os adolescentes. Cada um era incentivado a falar, perguntar e tocar nos métodos contraceptivos, como forma de familiarizar-se e favorecer o aprendizado. No espaço para falar exclusivamente dos métodos, os mais comentados entre eles, destacaram-se o DIU, as pílulas e a camisinha masculina, relacionando-as com as DST e a prevenção da gravidez.

Para finalizar as etapas da atividade educativa e observar a aprendizagem dos adolescentes sobre dúvidas que tinham sinalizado propôs-se a dinâmica: mitos e verdades sobre práticas de iniciação sexual e a contracepção. Os participantes responderam e discutiram coletivamente as questões apresentadas e percebeu-se que ainda tinham muitas dúvidas e incertezas sobre esta prática. Principais dúvidas evocadas pelos adolescentes na dinâmica e síntese das respostas dos facilitadores:

"O que é coito interrompido? É quando o homem retira o pênis antes de ejacular dentro da mulher. Considerado método de prevenção de gravidez, porém de pouca eficácia, porque na hora da relação sexual, o homem está muito excitado e é muito difícil ele querer retirar o pênis. Além disso, o líquido lubrificante que sai antes da segunda ejaculação contém poucos espermatozoides, logo a mulher pode engravidar".

"Por que o preservativo feminino é menos utilizado? Pela praticidade, é mais fácil encontrar a masculina na farmácia; pela colocação, tem que introduzir lá dentro da vagina e proteger o colo do útero; estética e conforto, porque pensam que o homem acha feio, e sentem menos prazer; a camisinha feminina é grande, as mulheres não gostam de colocar e o preço é mais elevado em comparação à camisinha masculina".

"Como se previne do HIV? A principal prevenção é utilizar camisinha durante as relações sexuais".

"Quando a menina tem relação sexual pela primeira vez, ela sangra? Não obrigatoriamente, porque existem cinco tipos de hímen, os com orificios maiores e menores e sem orificio que é preciso uma cirurgia para que ocorra a relação sexual. O que tem orifício maior, geralmente não sangra”. 
"Esses meninos que se masturbam prejudicam a saúde? Estudos mostram exatamente o oposto, diminui: o risco de infecção urinária e cervical, o risco de câncer de próstata, a insônia, o estresse, a dor e aumenta a força do pavimento pélvico".

A atividade educativa propiciou aos adolescentes questionarem, esclarecerem dúvidas acerca da prevenção da gravidez e métodos contraceptivos, no contexto da adolescência. Os facilitadores ao dialogarem com os adolescentes utilizaram linguagem clara e objetiva com bases científicas, essencialmente durante as dinâmicas; propiciando abordagem interativa, na tentativa de reconhecer os adolescentes em sua diversidade, assim como fortalecer ações de cuidado à saúde ${ }^{(16,25)}$.

Percebe-se, portanto, que as dúvidas relacionaram-se, em maior parte, com os métodos contraceptivos e a sua relação com o corpo, destacando-se as vozes das meninas, quando se questionavam sobre os efeitos anticoncepcionais orais e injetáveis, a camisinha feminina e sua forma de utilização, dentre outras ${ }^{(27)}$. A tentativa de esclarecer tais pontos e articular aos conhecimentos que já tinham, foi possível quando, na dinâmica, os facilitadores apresentaram os métodos contraceptivos por meio de materiais ilustrativos: banner e mostruário (preservativo masculino e feminino, DIU, diafragma, anticoncepção de emergência, espermicida, implante subcutâneo, anticoncepcional oral e injetável). Os adolescentes procuravam manusear e à medida que as dúvidas eram respondidas, havia apropriação das informações. Acredita-se que nesse momento houve um repensar de atitudes e práticas sobre a saúde sexual e a prevenção de uma gravidez não desejada para o momento.

Estudos realizados com adolescentes evidenciam que, a maioria deles, que são sexualmente ativos, não tem atitudes favoráveis à contracepção e os autores alertam que a abordagem sobre os métodos contraceptivos deve ser anterior à iniciação sexual ${ }^{(14,20)}$. Na presente pesquisa, observou-se que os métodos mais conhecidos entre eles era a camisinha e a pílula, porém as dúvidas eram constantes sobre as pílulas diárias (anticoncepcionais orais) e as pílulas do dia seguinte (anticoncepcionais de emergência). Os anticoncepcionais de emergência são métodos alternativos de anticoncepção para serem usados em situações especiais e não devem ser manipulados de forma contínua e diária ${ }^{(24)}$. Entretanto, muitos adolescentes desconhecem ou, mesmo sendo informado sobre o assunto, usam erroneamente.

Estudo $^{(24)}$ revelou que parte dos adolescentes nunca participou de conversas sobre a anticoncepção de emergência nas escolas e referiu ter informação de forma esporádica na escola pelo professor de Ciências. A escola é um ambiente propício para os adolescentes obterem estas informações e certamente envolverá atividades integradas com os profissionais da saúde, pois os educadores, na maioria das vezes, não estão informados sobre o assunto. No espaço da atenção básica, também, devem ser propiciadas estas ações na integração com a escola, um ambiente para muitos aprendizados, mas não ocorre a devida articulação entre estes setores, apesar da existência de programas com esta finalidade ${ }^{(10)}$.

Observa-se que embora ocorram atividades educativas na escola com os adolescentes, mas os educadores ainda têm muitas restrições ao discutir a temática sexualidade e contracepção. Preconize-se que a saúde e a educação devem atuar integrados na educação sexual dos adolescentes, mas no cotidiano há distanciamento desta realidade e eles tornam-se cada vez mais vulneráveis em adquirir DST/HIV/AIDS ou de ter uma gravidez não planejada ${ }^{(11)}$. Nesse contexto, a experiência desta intervenção indica a necessidade de elaborar e executar novas propostas para educação em saúde reprodutiva nas escolas e nos serviços de saúde, que alcancem os adolescentes antes do início da vida sexual, para que possam escolher a melhor forma de prevenir uma gravidez não planejada e infecções sexualmente transmissíveis.

\section{CONSIDERAÇÕES FINAIS}

A pesquisa em sua etapa exploratória utilizou pressupostos da pesquisa-ação, proporcionando participação dos adolescentes a partir de questionamentos e de reflexões sobre métodos contraceptivos e gravidez na adolescência. Assim, no desenvolvimento das dinâmicas promoveramse ações educativas e, simultaneamente, apreenderam-se informações para a pesquisa.

Nesta perspectiva, observou-se que o conhecimento adquirido pelos adolescentes nesta temática, advém de interações sociais, principalmente com os pares e denotam falta de conversas mais direcionadas sobre os meios de prevenção, seja com os pais, nas escolas ou nas unidades de saúde. Apesar de os adolescentes conhecerem os métodos contraceptivos, comumente utilizados por eles, não há segurança e confiabilidade em relação ao uso do preservativo masculino, como também na anticoncepção de emergência, anticoncepcional oral e injetável, uma vez que suas afirmações mostravam esta realidade.

Desse modo, as oficinas e as rodas de conversas trouxeram esclarecimentos e redução de inquietações e a autorreflexão sobre a prevenção da gravidez, contribuindo na autonomia dos escolares ante a escolha dos contraceptivos. Sugere-se continuar dialogando com este público de forma a captar suas necessidades e curiosidades neste campo da saúde sexual e reprodutiva. 


\section{AGRADECIMENTOS}

Aos educadores que permitiram e cederam o espaço da sala de aula para a efetivação da pesquisa.

\section{REFERÊNCIAS}

1. World Health Organization (WHO). Health for the world's adolescents: a second chance in the second decade [Internet]. 2014 [acess 2015 Sep 01]. Available from: http://www.who.int/maternal_child_adolescent/ documents/second-decade/en/.

2. Nothaft SCS, Zanatta EA, Brumm MLB, Galli KSB, Erdtmann BK, Buss E et al. Sexualidade do adolescente no discurso de educadores: possibilidades para práticas educativas. Rev Min Enferm. 2014;18(2): 284-89

3. Oliveira JM, Oliveira JPM, Silva SS, Silva SS, Maracajá PB. Gravidez na adolescência: realidade e repercussões sobre atividade sexual. INTESA - Informativo Técnico do Semiárido (Pombal-PB). 2015; 9(2):16-22.

4. Silva MRB, Santos ME, Silva LA, Figueiredo Filho V; Maturana HCA. Porque elas não usam?: um estudo sobre a não adesão das adolescentes ao preservativo e suas repercussões. Saúde em Redes. 2015; 1(4): 75 83

5. Gondim PS, Souto NF, Moreira CB, Cruz MEC, Caetano FHP, Montesuma FG. Accessibility of adolescents to sources of information on sexual and reproductive health. Journal of Human Growth and Development. 2015; 25(1): 50-3

6. Spindola T, Ribeiro KS, Fonte VRF. A vivência da gravidez na adolescência: contribuições para a enfermagem obstétrica. Adolesc. Saude. 2015; 12 (1): 50-6.

7. Guerreiro EM, Rodrigues DP, Queiroz ABA, Ferreira MA. Health education in pregnancy and postpartum: meanings attributed by puerperal women. Rev. bras. enferm. [Internet]. 2014 [acess 2016 Oct 02]; 67(1):13-21. Available from: http://www. scielo.br/scielo.php?script $=$ sci_arttext\&pid $=$ S0034$71672014000100013 \& \operatorname{lng}=$ en.

8. Silva MMS, Dias MSA, Vasconcelos ACM, Souza DR, Mesquita JS. "Eu só quero amar": ação intersetorial pet-saúde/Programa saúde na escola com adolescentes. SANARE, Sobral. 2016; 15 (1): 90-7.

9. Higa EFR, Bertolin FH, Maringolo LF, Ribeiro TFSA, Ferreira LHK, Oliveira VASC. A intersetorialidade como estratégia para promoção da saúde sexual e reprodutiva dos adolescentes. Interface Comunicação Saúde Educação. 2015; 19 Supl 1:879-91

10. Brasil. Ministério da Saúde. Secretaria de Atenção em Saúde. Departamento de Ações Programáticas Estratégicas. Diretrizes nacionais para a atenção integral à saúde de adolescentes e jovens na promoção, proteção e recuperação da saúde. / Ministério da Saúde, Secretaria de Atenção em Saúde, Departamento de Ações Programáticas Estratégicas, Área Técnica de Saúde do Adolescente e do Jovem. - Brasília: Ministério da Saúde; 2010.

11. Rodrigues MGS, Cosentino SF, Rossetto M, Maia KM, Pautz M, Silva VC. Oficinas educativas em sexualidade do adolescente: a escola como cenário. Enfermería Global: revista electrônica quatrimestral de Enfermería. 2010; (20), 1-8. Disponível em: http:// scielo.isciii.es/pdf/eg/n20/pt_docencia2.pdf. Acesso em 18de setembro 2016.

12. Brasil. Ministério da Saúde. Ministério da Educação. Guia de sugestões de atividades: Semana Saúde na Escola. Tema: Sexualidades e Saúde Reprodutiva. Brasília. Distrito Federal; 2013.

13. Araújo RLD, Nóbrega AL, Nóbrega JYL, Silva G, Sousa KMO, Coelho DC et al. Gravidez na adolescência: consequências voltadas para a mulher. INTESA Informativo Técnico do Semiárido (Pombal-PB). 2015; 9(1): 15-22.

14. Gonçalves H, Machado EC, Soares ALG, CamargoFiguera FA, Seerig LM, Mesenburg MA et al . Início da vida sexual entre adolescentes (10 a 14 anos) e comportamentos em saúde. Rev. Bras Epidemiol. [Internet]. 2015 [acess 2016 Oct 18]; 18(1): 25-41. Available from: http://www.scielo.br/ scielo.php?script=sci_arttext\&pid=S1415$790 \times 2015000100025 \& \operatorname{lng}=$ en.

15. Thiollent M. Metodologia da Pesquisa-Ação. (17 edição). São Paulo: Cortez; 2011.

16. Priotto EP. Dinâmicas de grupos para adolescentes. Petrópolis: Vozes; 2008.

17. Minayo MCS. O desafio do conhecimento: pesquisa qualitativa em saúde. São Paulo: Hucitec; 2010.

18. Brasil. Resolução 466 de 12 de dezembro de 2012. Dispõe sobre as diretrizes e normas regulamentadoras de pesquisas envolvendo seres humanos. Plenário do Conselho Nacional de Saúde. Brasília, DF, 12 de dez. de 2012; 2012.

19. Duarte CF, Holanda LB, Medeiros ML. Avaliação de conhecimento contraceptivo entre adolescentes 
grávidas em uma unidade básica de saúde do Distrito Federal. J Health Sci Inst. 2012; 30(2): 140-3.

20. Costa GPO, Guerra AQS, Araújo ACPF. Conhecimentos, atitudes e práticas sobre contracepção para adolescentes. J. res.: fundam. care. Online. 2016; 8(1): 3597-608.

21. Malta DC, Silva MAI, Mello FCM, Monteiro RA, Porto DL, Sardinha LMV et al . Saúde sexual dos adolescentes segundo a Pesquisa Nacional de Saúde dos Escolares. Rev Bras. epidemiol. [Internet]. 2011 [acess 2016 Oct 18]; 14 (Suppl 1): 147-156. Available from: http://www.scielo. br/scielo.php?script $=$ sci_arttext\&pid $=$ S1415790X2011000500015\&lng=en.

22. Koerich MS, Baggio MA, Backes MTS, Backes DS, Carvalho JN, Meirelles BHS et al. Sexualidade, doenças sexualmente transmissíveis, e contracepção: atuação da enfermagem com jovens da periferia. Rev. Enferm. UERJ. 2010; 18(2): 265-71.

23. Dias ACG, Teixeira MAP. Gravidez na adolescência: um olhar sobre um fenômeno complexo. Paidéia. Ribeirão Preto. 2010; 20(45): 123-31.
24. Chofakian CBN, Borges ALV, Fujimori E, Hoga LAK. Conhecimento sobre anticoncepção de emergência entre adolescentes do Ensino Médio de escolas públicas e privadas. Cad. Saúde Pública. 2014; 30(7): 1525-36.

25. Costa MMA, Frare JC, Nobre JRS, Tavares KO. A maternidade e a paternidade: o olhar do casal adolescente. Rev Bras Promoç Saúde. 2014; 27(1): 101-08.

26. Manfré CC, Queiróz SG, Matthes ACS. Considerações atuais sobre gravidez na adolescência. R. bras. Med. Fam. e Comun. 2010; 5(17): 48-54.

27. Soares LR, Cabero FV, Souto TG, Coelho RFS, Lacerda LCM, Matão MEL. Avaliação do comportamento sexual entre jovens e adolescentes de escolas públicas. Adolesc. Saúde. 2015; 12 (2): 76-84.

28. Fiedler M, Araujo WA, Souza MCC.A prevenção da gravidez na adolescência na visão de Adolescentes. Texto Contexto Enferm. 2015; 24 (1): 30-7.

\section{Endereço para correspondência:}

Maria Veraci Oliveira Queiroz

Universidade Estadual do Ceará - UECE

Avenida Dr. Silas Muguba, 1700 - Campus do Itaperi

CEP: 60740.000 - Fortaleza - CE - Brasil

E-mail: veracioq@hotmail.com 